Pacific Journal of Mathematics

ON THE SOLVABILITY OF NONLINEAR FUNCTIONAL 


\title{
ON THE SOLVABILITY OF NONLINEAR FUNCTIONAL EQUATIONS OF 'MONOTONIC' TYPE
}

\author{
George J. MinTy
}

1. Introduction. Let $X$ and $Y$ be a conjugate pair of reflexive Banach spaces (with real or complex scalars) such that $X$ has a smooth unit ball. For $x \in X, y \in Y$, we denote the natural pseudo-inner-product by $\langle x, y\rangle$. Let $f: X \rightarrow Y$ be a continuous monotonic function-i.e., one satisfying $R e\left\langle x_{1}-x_{2}, f\left(x_{1}\right)-f\left(x_{2}\right)\right\rangle \geqq 0$ for all $x_{1}, x_{2} \in X$. The main object of this paper is to present a theorem on the solvability of the equation $f(x)=u$, for given $u \in Y$, analogous to the ordinary "intermediatevalue theorem" for a continuous (monotonic!) real-valued function of a real variable. In finite-dimensions, the known result [1] is that the range $R$ of $f$ is an almost-convex set (contains the interior of its convex hull, where "interior" may be taken relative to the smallest real flat containing $R$--see below for the definitions).

In order to preserve, so far as possible, duality between the domain and the range of $f$, theorems will be proved first on "monotonic" subsets of the product-space $X \times Y$, and then afterwards applied to the graph of $f$.

The theorems of this paper result from an attempt to obtain the same general kind of theorems as one gets by the "variational method", as developed especially by $\mathrm{E}$. $\mathrm{H}$. Rothe, without assuming that $f$ is the Fréchet differential of a real scalar function. In the variational theory, the assumption of monotonicity of $f$ turns up in the form of convexity of the associated scalar, which in turn guarantees weak lower-semicontinuity. (In order to see the connection, compare Theorem 6 of [3] and Theorem 4.2 of [6]).

2. Preliminaries. Let $X$ be a Banach-space and $Y$ its conjugatespace, or vice versa. In $X \times Y$, we define the $M$-relation (as in [1], [3]) by: $\left(x_{1}, y_{1}\right) M\left(x_{2}, y_{2}\right)$ provided $R e\left\langle x_{1}-x_{2}, y_{1}-y_{2}\right\rangle \geqq 0$. A set $E \subset(X \times Y)$ is called monotonic provided each pair of points of $E$ is $M$-related, and is called maximal if it cannot be embedded in a properly larger monotonic subset of $X \times Y$. If, for any $\left(x_{1}, y_{1}\right)$ and $\left(x_{2}, y_{2}\right) \in E$ we have $R e\left\langle x_{1}-x_{2}, y_{1}-y_{2}\right\rangle=0$ implies $x_{1}=x_{2}$ and $y_{1}=y_{2}$, then $E$ will be called strictly monotonic.

Note that $\langle x, y\rangle$ is a bilinear form rather than a sesquilinear form, that is, for $\alpha$ complex, $\langle x, \alpha y\rangle=\alpha\langle x, y\rangle$. Nevertheless, in the theorems of this paper, it may be thought of as the usual inner product when $X=Y=H$, where $H$ is a Hilbert space, because the

Received February 11, 1963. 
statements of the theorems refer only to the functional value of $\langle x, y\rangle$, and not to the function itself.

For a set $S$ in a normed linear space, $K[S]$ denotes the convex hull of $S$. All topological terms refer to the norm-topology unless explicitly otherwise stated. The unit ball of a real Banach-space is called smooth provided that at each point of the boundary there is a unique hyperplane of support; sufficient conditions for this are that the norm be Fréchet-differentiable at all points of the boundary, or that the unit ball of the conjugate-space be strictly convex. The unit ball of a complex Banach-space is called smooth if the unit ball of the real Banach-space obtained by restricting the scalars to be real, is smooth. A real linear subspace is a linear subspace of this real Banach-space, in the complex case; in the real case, it is an ordinary linear subspace. A real flat is a translate of a closed real linear subspace. The symbols $P_{1}$ and $P$ refer to the projection-maps of a product space: $P_{1}(x, y)=x, P(x, y)=y$. The open sphere with center $u$ and radius $\varepsilon$ is called $S(\varepsilon ; u)$, and the closed sphere (ball) is called $\subseteq(\varepsilon ; u)$. The zero-vector of a linear space is called $\theta$.

\section{Uniqueness-theorems.}

THEOREM 1. Let $E \subset(X \times Y)$ be a maximal monotonic set, and $u \in Y$. Then $S=\{x:(x, u) \in E\}$ is a closed convex set. Moreover, if $E$ is strictly monotonic, then $S$ is at most a single point.

Proof. Let $\left(x_{1}, u\right),\left(x_{2}, u\right) \in E$, and let $s, t$ be positive real numbers with $s+t=1$. Let $\left(x_{0}, y_{0}\right)$ be any point of $E$. Then

$$
\begin{aligned}
& R e\left\langle\left(s x_{1}+t x_{2}\right)-x_{0}, u-y_{0}\right\rangle \\
& \quad=s R e\left\langle x_{1}-x_{0}, u-y_{0}\right\rangle+t R e\left\langle x_{2}-x_{0}, u-y_{0}\right\rangle \geqq 0
\end{aligned}
$$

so that $\left(s x_{1}+t x_{2}, u\right) M\left(x_{0}, y_{0}\right)$. Hence $\left(s x_{1}+t x_{2}, u\right) \in E$ by the maximality of $E$; it follows that $S$ is convex. To show that $S$ is closed, we put $Z=\{(x, u): x \in X\}$, and note that

$$
E=\bigcap_{\left(x_{0}, y_{0}\right) \in E}\left\{(x, y): R e\left\langle x-x_{0}, y-y_{0}\right\rangle \geqq 0\right\}
$$

and $Z$ are both closed subsets of $X \times Y$ (taken with the usual producttopology), so that $T=Z \cap E$ is a closed subset of $X \times Y$, and hence of $Z$. Now, there is an obvious homeomorphism of $Z$ onto $X$ which maps $T$ onto $S$; the closedness of $S$ follows.

The second statement of Theorem 1 is trivial.

The main purpose of presenting this rather elementary theorem is to exhibit that a maximal monotonic set has essentially the same uniqueness-properties as the graph of the Fréchet differential of a 
convex function, which is (broadly speaking) a special case.

4. Main existence-theorem. If $X$ is a complex Banach-space with conjugate-space $Y$, and $X^{\prime}$ is the corresponding real Banach-space obtained by restricting the scalars to be real, with conjugate-space $Y^{\prime}$, it is well known that $y^{\prime}=R e\langle\cdot, y\rangle$ is a norm-preserving isomorphism of $Y$ onto $Y^{\prime}$. It would be possible to prove the lemmas and theorem of this section first in the case of a real Banach-space, and then use this isomorphism to extend them to the complex case; however, we shall continue for the present to work simultaneously with the real and complex cases.

Lemma 1. Let $X$ be a Banach-space and $Y$ its conjugate-space, or vice-versa. Suppose $A \subset(X \times Y)$ is a monotonic set with the properties:

(i) $K[P(A)]$ contains a ball $\subseteq(\varepsilon ; \theta)$, with $\varepsilon>0$.

(ii) The set $\{R e\langle x, y\rangle:(x, y) \in A\}$ is bounded above by $M>0$. Then, for any $y_{0} \in Y$ with $\left\|y_{0}\right\|>M / \varepsilon$, there exists a finite subset $\left\{\left(x_{i}, y_{i}\right): i=1, \cdots, m\right\}$ of $A$ such that, with

$$
Q=\bigcap_{i=1}^{m}\left\{x: R e\left\langle x_{i}-x, y_{i}\right\rangle \geqq 0\right\},
$$

$x \in Q$ implies $R e\left\langle x, y_{0}\right\rangle<\left\|y_{0}\right\|^{2}$.

Proof. Consider any $y_{0}$ with $\left\|y_{0}\right\|>M / \varepsilon$. Then $\varepsilon y_{0}\left\|y_{0}\right\|$ lies in $\subseteq(\varepsilon ; \theta)$, and hence by (i) it is a convex combination of vectors of $P(A)$ :

$$
\varepsilon y_{0} /\left\|y_{0}\right\|=\lambda_{1} y_{1}+\cdots+\lambda_{m} y_{m} \quad\left(\lambda_{i}>0, I=\Sigma \lambda_{i}\right),
$$

Forming the pseudo-inner-product of both sides with any $x \in Q$ and then taking real parts, we see that

$$
\begin{aligned}
\frac{\varepsilon}{\left\|y_{0}\right\|} R e\left\langle x, y_{0}\right\rangle & =\Sigma \lambda_{i} R e\left\langle x, y_{i}\right\rangle \leqq \Sigma \lambda_{i} R e\left\langle x_{i}, y_{i}\right\rangle \\
& \leqq \Sigma \lambda_{i} M=M
\end{aligned}
$$

so that

$$
R e\left\langle x, y_{0}\right\rangle \leqq \frac{M\left\|y_{0}\right\|}{\varepsilon} \leqq\left\|y_{0}\right\|^{2}
$$

Lemma 2. With $X$ and $Y$ as in Lemma 1, let $\left\{\left(x_{i}, y_{i}\right): i=\right.$ $1, \cdots, m\}$ be any finite subset of $X \times Y$. Then the set

$$
R=\bigcap_{i=1}^{m}\left\{x: \operatorname{Re}\left\langle x_{i}-x, y_{i}\right\rangle \geqq 0\right\}
$$

is nonempty. 
Proof. The main theorem of [2] asserts the stronger statement that for any $y \in Y$, there exists $x$ satisfying $R e\left\langle x_{i}-x, y_{i}-y\right\rangle \geqq 0$ for all $i$. Actually, the theorem of [2] is stated for Hilbert spaces, so a short argument is necessary here, as follows:

If $Y$ is the conjugate-space of $X$ : let $X^{\prime}$ be the finite-dimensional linear subspace of $X$ generated by $x_{1}, \cdots, x_{m}$. Let $Y^{\prime}$ be the conjugatespace of $X^{\prime}$, and let $y_{i}^{\prime} \in Y^{\prime}$ be the restrictions of the functionals $y_{i}$ to $X^{\prime}$. Distinguishing a basis in $X^{\prime}$, one can now easily make a Hilbert space out of $X^{\prime}$ in the usual way, and identify $Y^{\prime}$ with $X^{\prime}$; the rest of the proof is simple.

If $X$ is the conjugate-space of $Y$ : let $Y^{\prime}$ be the finite-dimensional linear subspace generated by $y_{1}, \cdots, y_{m}$, and let $X^{\prime}$ be its conjugatespace. Let $x_{i} \in X^{\prime}$ be the restrictions of the $x_{i}$ to $Y^{\prime}$. Impose a Hilbert-space structure on $Y^{\prime}$, identify $X^{\prime}$ with $Y^{\prime}$, and construct $x^{\prime}$ in $X^{\prime}$ by the theorem of [2]. Now let $x$ be any Hahn-Banach extension of $x^{\prime}$ to all of $X$.

Lemma 3. Now let $X$ be a Banach-space and $Y$ be its conjugatespace, and assume that the unit ball of $X$ is smooth. Suppose the monotonic set $E \subset(X \times Y)$ has a subset $A$ satisfying (i) and (ii) of Lemma 1. Let $\left(x_{1}, y_{1}\right), \cdots,\left(x_{n}, y_{n}\right)$ be any finite subset of $E$, and let $R=\bigcap_{i=1}^{n}\left\{x: R e\left\langle x_{i}-x, y_{i}\right\rangle \geqq 0\right\}$. Then $R$ contains a point $x_{0}$ having $\left\|x_{0}\right\| \leqq M / \varepsilon$.

Proof. By Lemma 2, $R$ is nonempty. Now, $R$ is convex and closed, so (as is well known) the norm in $X$ assumes its minimum on $R$ at a point $x_{0}$. Suppose $\left\|x_{0}\right\|>M / \varepsilon$. We take up first the case where the scalars are real.

By the Hahn-Banach theorem, there exists $y_{0} \in Y$ such that $\left\langle x_{0}, y_{0}\right\rangle=\left\|x_{0}\right\|^{2}$, and $\left\|y_{0}\right\|=\left\|x_{0}\right\|$. (Note that by the smoothness of the unit ball in $X$, there is only one $y_{0}$ satisfying these conditions.) Since $\left\|y_{0}\right\|>M / \varepsilon$, Lemma 1 asserts that there exists $\left(x_{n+1}, y_{n+1}\right), \cdots$, $\left(x_{n+m}, y_{n+m}\right)$ in $A$, and hence in $E$, such that, with

$$
Q=\bigcap_{i=n+1}^{n+m}\left\{x:\left\langle x_{i}-x, y_{i}\right\rangle \geqq 0\right\},
$$

$x \in Q$ implies that $\left\langle x, y_{0}\right\rangle\left\langle\left\|y_{0}\right\|^{2}\right.$, and thus that $\left\langle x, y_{0}\right\rangle\left\langle\left\langle x_{0}, y_{0}\right\rangle\right.$. Now, by Lemma $2, R \cap Q$ is nonempty; say, $x_{1} \in R \cap Q$. Thus

$$
\left\langle x_{1}, y_{0}\right\rangle<\left\langle x_{0}, y_{0}\right\rangle
$$

and $x_{1} \neq x_{0}$.

Now, if $x_{1}$ is linearly dependent on $x_{0}$, then $x_{1}=c x_{0}$ for some real $c$, so that $c\left\langle x_{0}, y_{0}\right\rangle\left\langle\left\langle x_{0}, y_{0}\right\rangle\right.$, and $c<1$ (since $\left\langle x_{0}, y_{0}\right\rangle>0$ ). Since $R$ is convex, it contains the line-segment joining $x_{0}$ and $x_{1}$, so $x_{0}$ could 
not be an element of $R$ with smallest norm. On the other hand, if $x_{1}$ and $x_{0}$ are linearly independent, we can apply the Hahn-Banach theorem to obtain $y_{1} \in Y$ such that $\left\langle x_{1}, y_{1}\right\rangle=\left\|x_{1}\right\| \cdot\left\|x_{0}\right\|$, and $\left\langle x_{0}, y_{1}\right\rangle=$ $\left\|x_{0}\right\|^{2}$, and $\left\|y_{1}\right\|=\left\|x_{0}\right\|$. By the smoothness of the unit ball, $y_{1}=y_{0}$, and hence from $(*),\left\|x_{1}\right\| \cdot\left\|x_{0}\right\|<\left\|x_{0}\right\|^{2}$ and $\left\|x_{1}\right\|<\left\|x_{0}\right\|$, again contradicting the minimality of the norm of $x_{0}$ over $R$. This completes the proof when the scalars are real.

If the scalars are complex, we make appeal to the earlier-mentioned norm-preserving isomorphism between the conjugate-spaces of the complex Banach-space $X$ and the corresponding real Banach-space $X^{\prime}$, and the conclusion is immediate.

Theorem 2. (Main Existence-Theorem) Let $X$ and $Y$ be a conjugate pair of reflexive Banach-spaces such that $X$ has smooth unit ball, and suppose $E \subset(X \times Y)$ is a maximal monotonic set. Then a sufficient condition for $\theta \in P(E)$ is that there exist a set $A \subset E$ with the properties:

(i) $\theta \in$ int $K[P(A)]$

(ii) $\{R e\langle x, y\rangle:(x, y) \in A\}$ is bounded above.

Proof. Let $\varepsilon>0$ be such that $\subseteq(\varepsilon: \theta) \subset K[P(A)]$, and $M>0$ be such that $\{R e\langle x, y\rangle:(x, y) \in A\}<M$. Let the points of $E$ be indexed by $\alpha$, so that $E=\left\{\left(x_{\alpha}, y_{\alpha}\right)\right\}$. Consider the sets

$$
T_{a}=\left\{x: R e\left\langle x_{a}-x, y_{a}\right\rangle \geqq 0,\|x\| \leqq M / \varepsilon\right\} .
$$

By Lemma 3, the intersection of any finite subcollection of these sets is nonempty. Since the $T_{\infty}$ are all weakly-closed subsets of the weaklycompact ball $\Im(M / \varepsilon ; \theta)$, it follows (from the "finite-intersection property" of compact sets) that the intersection of $\subseteq(M / \varepsilon ; \theta)$ and all the $T_{a}$ is nonempty: say, it contains $x$. Thus for every $\alpha,(x, \theta) M\left(x_{\alpha}, y_{\alpha}\right)$, and since $E$ is maximal, $(x, \theta) \in E$ and $\theta \in P(E)$.

5. Generalizations of the main theorem. The main theorem generalizes easily in many directions simultaneously, so that a very general, but unwieldy, main theorem could be stated. The writer feels that it is best merely to indicate the directions of such generalizations. To keep the discussion lucid, we shall discuss only the special case where the maximal monotonic set $E$ is the graph of a monotonic function $f: X \rightarrow Y$. (See Paragraph 6.)

REMARK 1. It is easily seen, from the proof of Theorem 2, that an upper bound on the norm of the solution of $f(x)=\theta$ constructed 
there, is given by $\|x\| \leqq M / \varepsilon$. It is not hard to sharpen this upper bound to $M_{0} / \varepsilon_{0}$, where $M_{0}$ is the g.l.b. of all possible $M$, and $\varepsilon_{0}$ is the l.u.b. of all possible $\varepsilon$, by applying Theorem 2 (or rather, the proof of the theorem) to the sequence of pairs $\left(M_{1}, \varepsilon_{1}\right),\left(M_{2}, \varepsilon_{2}\right), \cdots$, where $M_{i}$ is a decreasing sequence approaching $M_{0}$ and $\varepsilon_{i}$ is an increasing sequence approaching $\varepsilon_{0}$, and using the weak compactness of $\subseteq\left(N_{1} / \varepsilon_{1} ; \theta\right)$. If the unit ball of $X$ is not smooth, an equivalent norm may sometimes be introduced to smooth it. But note that introduction of an equivalent norm changes not only the form of the inequality $\|x\| \leqq$ $M / \varepsilon$, but may also effect a change in $\varepsilon$, since the norm in $Y$ must be changed correspondingly.

REMARK 2. To prove the existence of a solution of the equation $f(x)=u$, with $u \neq \theta$, one should work with the function $g(x)=$ $f(x)-u$, or even with $g(x)=f(x-v)-u$, with a judicious choice of $v$.

REMARK 3. The existence of a solution of $x+f(x)=u$, for monotonic $f$, has already been shown elsewhere [3]. The theorems of [3] can be generalized to solve the equation $\lambda x+f(x)=u$, for $\operatorname{Re} \lambda>0$, by the use of the map $\phi(x, y)=(\lambda x+y, \bar{\lambda} x-y)$ in place of the map $\phi$ of that paper.

REMARK 4. If the entire range of $f$ is contained in a closed linear proper subspace $Y_{0}$ of $y$, then it is impossible to satisfy (i) of Theorem 2. But virtually the same trick can be used as in [1]: note that $f$ must be constant on any coset of $X \bmod Y_{0}^{\perp}$, the orthogonal complement of $Y_{0}$, so that $f$ can be regarded as a function mapping $X / Y_{0}^{\perp}$ into $Y_{0}$, this function is still monotonic. Also, by a standard argument, $X / Y_{0}^{\perp}$ and $Y_{0}$ are a conjugate pair of reflexive Banachspaces, and the unit ball of $X / Y_{0}$ is smooth if that of $X$ is. Also: it is easily seen, by the discussion given in [1], that one can work with the underlying real Banach-space of $X$ and take $Y_{0}$ as the smallest real closed linear subspace containing the range of $f$.

This trick corresponds to taking the "interior," in the statement of Theorem 2, relative to the smallest closed real linear subspace containing the range of $f$. In case one wishes to solve the equation $f(x)=u$, as in Remark 2, the interior is taken relative to the smallest real flat containing the range of $f$.

6. An application. Let $H$ be a Hilbert space; let $f: H \rightarrow H$ have the property that its Fréchet differential exists everywhere and is a "dissipative" linear operator:

$$
R e\left\langle\Delta x, f^{\prime}(x ; \Delta x)\right\rangle \leqq 0 \quad \text { for all } x, \Delta x,
$$


and that the range of $f$ lies entirely in some closed linear subspace $H_{0} \subset H$. Then we can state the following:

THEOREM 3. A sufficient condition for $f(x)=\theta$ to have a solution is: there exists a set $B \subset H$ such that and

(i) $\theta \in \operatorname{int} K[P(f(B))]$ (interior relative to $H_{0}$ )

(ii) the set $\{R e\langle x, f(x)\rangle: x \in B\}$ is bounded below. The solution is unique if $f^{\prime}$ is strictly dissipative.

Proof. Regard $f$ as a monotonic function from $H_{0}$ into $H_{0}$, as in Remark 4. Let $g(x)=-f(x)$. Then $g$ has an "accretive" Fréchet differential. By Therem 6 of [3], the graph of $g$ is a maximal monotonic set in $H_{0} \times H_{0}$. Theorem 2 above completes the existence-proof, and an adaptation of the proof of Theorem 6 of [3] shows that if $g^{\prime}$ is strictly accretive, then $g$ is strictly monotonic; Theorem 1 of the present paper completes the uniqueness proof.

Theorem 3 can be generalized to some cases in which $f$ is not everywhere differentiable, or even everywhere-defined; see [3], Theorems 4 and 5 .

7. Acknowlegement. The author wishes to thank Professor E. H. Rothe for many helpful conversations, and to remark that the present paper has been much influenced, in non-obvious ways, by his earlier papers [4] and [5].

8. Added in proof. A close relative of the theorem of this paper will appear in anotner paper of the writer in Proc. Nat. Acad. Sciences (U.S.A).

\section{REFERENCES}

1. G. J. Minty, On the maximal domain of a 'monotone' function, Mich. Math. J., 8 (1961), 125-137.

2. - On the simultaneous solution of a certain system of linear inequalities, Proc. Amer. Math. Soc., 13 (1962), 11-12.

3. - Monotone (nonlinear) operators in Hilbert space, Duke Math. J., 29 (1962), $341-346$.

4. E. H. Rothe, Gradient mappings and extrema in Banach spaces, Duke Math. J., 15 (1948), 421-431.

5. — Gradient mappings, Bull. Amer. Math. Soc., 59 (1953), 5-19.

6. - A note on the Banach spaces of Calkin and Morrey, Pacific J. Math., 3 (1953), 493-499. 



\section{PACIFIC JOURNAL OF MATHEMATICS}

\section{EDITORS}

Robert Osserman

Stanford University

Stanford, California

M. G. Arsove

University of Washington

Seattle 5 , Washington
J. DugundjI

University of Southern Califorma: Los Angeles 7, California

Lowell J. Paige

University of California

Los Angeles 24, California

\section{ASSOCIATE EDITORS}

E. F. BECKENBACH

B. H. NeumanN

F. WOLF

K. YosIDA

\section{SUPPORTING INSTITUTIONS}

UNIVERSITY OF BRITISH COLUMBIA

CALIFORNIA INSTITUTE OF TECHNOLOGY

UNIVERSITY OF CALIFORNIA

MONTANA STATE UNIVERSITY

UNIVERSITY OF NEVADA

NEW MEXICO STATE UNIVERSITY

OREGON STATE UNIVERSITY

UNIVERSITY OF OREGON

OSAKA UNIVERSITY

UNIVERSITY OF SOUTHERN CALIFORNIA
STANFORD UNIVERSITY

UNIVERSITY OF TOKYO

UNIVERSITY OF UTAH

WASHINGTON STATE UNIVERSITY

UNIVERSITY OF WASHINGTON

AMERICAN MATHEMATICAL SOCIETY CALIFORNIA RESEARCH CORPORATION SPACE TECHNOLOGY LABORATORIES NAVAL ORDNANCE TEST STATION 


\section{Pacific Journal of Mathematics}

\section{Vol. 14, No. 1 \\ May, 1964}

Richard Arens, Normal form for a Pfaffian .........................

Charles Vernon Coffman, Non-linear differential equations on cones in Banach

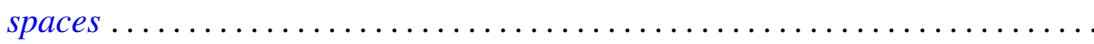

Ralph DeMarr, Order convergence in linear topological spaces ..............

Peter Larkin Duren, On the spectrum of a Toeplitz operator ................

Robert E. Edwards, Endomorphisms of function-spaces which leave stable all

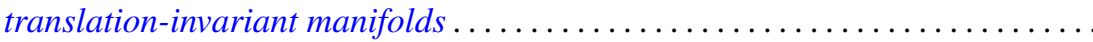

Erik Maurice Ellentuck, Infinite products of isols . . . . . . . . . . . . . . . . 49

William James Firey, Some applications of means of convex bodies . . . . . . . . 53

Haim Gaifman, Concerning measures on Boolean algebras ............. 61

Richard Carl Gilbert, Extremal spectral functions of a symmetric operator. . . . . . 75

Ronald Lewis Graham, On finite sums of reciprocals of distinct nth powers ..... 85

Hwa Suk Hahn, On the relative growth of differences of partition functions ...... 93

Isidore Isaac Hirschman, Jr., Extreme eigen values of Toeplitz forms associated

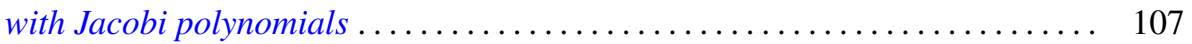

Chen-jung Hsu, Remarks on certain almost product spaces . . . . . . . . . . . 163

George Seth Innis, Jr., Some reproducing kernels for the unit disk . . . . . . . . . 177

Ronald Jacobowitz, Multiplicativity of the local Hilbert symbol . . . . . . . . . . . 187

Paul Joseph Kelly, On some mappings related to graphs ................. 191

William A. Kirk, On curvature of a metric space at a point . . . . . . . . . . . . 195

G. J. Kurowski, On the convergence of semi-discrete analytic functions . . . . . . . 199

Richard George Laatsch, Extensions of subadditive functions . . . . . . . . . . . 209

V. Marić, On some properties of solutions of $\Delta \psi+A\left(r^{2}\right) X \nabla \psi+C\left(r^{2}\right) \psi=0 \ldots 217$

William H. Mills, Polynomials with minimal value sets . . . . . . . . . . . 225

George James Minty, Jr., On the monotonicity of the gradient of a convex

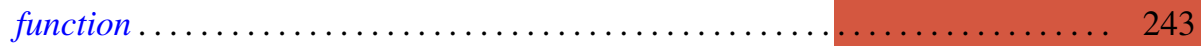

George James Minty, Jr., On the solvability of nonlinear functional equations of 'monotonic' type ................................... 249

J. B. Muskat, On the solvability of $x^{e} \equiv e(\bmod p) \ldots \ldots \ldots \ldots \ldots \ldots \ldots \ldots . \ldots \ldots$

Zeev Nehari, On an inequality of $P . R$. Bessack ................... 261

Raymond Moos Redheffer and Ernst Gabor Straus, Degenerate elliptic

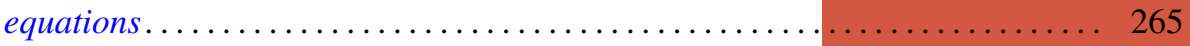

Abraham Robinson, On generalized limits and linear functionals . . . . . . . . . 269

Bernard W. Roos, On a class of singular second order differential equations with a

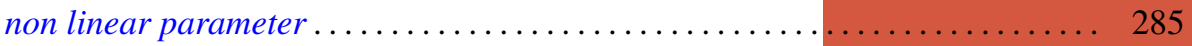

Tôru Saitô, Ordered completely regular semigroups . . . . . . . . . . . . . . . . 295

Edward Silverman, A problem of least area ....................... 309

Robert C. Sine, Spectral decomposition of a class of operators . . . . . . . . . 333

Jonathan Dean Swift, Chains and graphs of Ostrom planes . . . . . . . . . . . 353

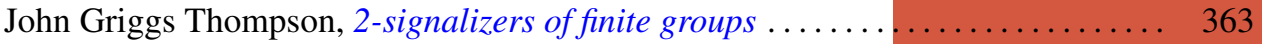

Harold Widom, On the spectrum of a Toeplitz operator . . . . . . . . . . . . . 365 